\title{
Implant salvage in an irradiated breast reconstruction patient with periprosthetic infection and implant exposure
}

\author{
Alexandru Daniel Hernic ${ }^{2}$, Iulia Cristina Filip², Ioana Dogaru², Silviu Constantin \\ Badoiu $^{1,2}$, Cristian Radu Jecan*,1,2
}

1"Carol Davila" University of Medicine and Pharmacy, Bucharest, Romania, ${ }^{2}$ Department of Plastic and Reconstructive Surgery, "Prof. Dr. Agrippa Ionescu" Clinical Emergency Hospital, Bucharest, Romania

\begin{abstract}
Analysing the trends over the last 2 decades, we can observe a steady increase in the percentage of women who choose to undergo breast reconstruction procedures following mastectomy. This is indisputably attributed to the high quality modern breast cancer multimodality treatment protocols, which currently allow not only for achieving a disease-free status but also for improving on patients' general well-being by restoring one's image and self-esteem. Case report. A 55 year-old non-smoking, former breast cancer female patient, presented to our clinic, in full accordance with the oncological department, requesting a unilateral breast reconstruction procedure. Patient's history revealed a multimodality treatment comprising radio-, chemo- and hormonotherapy following mastectomy. After having signed the informed consent, the patient opted for a two-stage expander-implant reconstruction. Six weeks after expander replacement the patient presented with sudden onset cellulitis on the reconstructed breast accompanied by wound dehiscence and minor implant exposure. Implant salvage was attempted, initially through additional flap dissection and readvancement, afterwards by means of a myocutaneous latissimus dorsi flap and later on in an acceptable manner through a fasciocutaneous locoregional perforator flap using infrared thermal imaging. The reconstructive procedure will be resumed 6 months after surgery when nipple-areolar complex reconstruction and contralateral symmetrisation mammaplasty are envisaged. Conclusion. Whilst a large array of breast reconstruction options are currently available, it is impossible to recur to a one-size fits all procedure from which all breast cancer patients may benefit. Adequate patient selection is regarded as a major contributing factor to overall procedure success.
\end{abstract}

Keywords: breast reconstruction, implant salvage, thermal imaging, periprosthetic infection, multimodality treatment, expander-based reconstruction, latissimus dorsi

\section{Introduction}

Analysing the trends over the last 2 decades, a steady increase in the percentage of women who choose to undergo breast reconstruction procedures following mastectomy can be observed [1, 2]. This is indisputably attributed to the high quality

Received: October 2015; Accepted after review: December 2015; Published: December 2015.

${ }^{*}$ Corresponding author: Jecan Radu MD PhD, Department of Plastic and Reconstructive Surgery, "Prof. Dr. Agrippa Ionescu" Clinical Emergency Hospital, 7 Ion Mincu St., Postal Code 011356, Sect. 1, Bucharest, Romania

Email: radu.jecan@gmail.com modern breast cancer multimodality treatment protocols [3], which currently allow not only for achieving a disease-free status but also for improving on patients' general well-being by restoring one's image and self-esteem [4].

Whilst we are presently witnessing a large array of breast reconstruction options, each with its own advantages and disadvantages [5], it is impossible to pin point a one-size fits all procedure from which all breast cancer patients may benefit [6], rendering adequate patient selection as a major contributing factor to overall procedure success [7-9].

Seeing that the patient is to be included in the decision-making process, appropriate patient education is not only to be envisaged 
but rather mandatory $[7,10]$. Unrealistic expectations must be tempered [10] and jointly acknowledged objectives concerning breast's shape and volume, possible symmetry and reconstruction of the nipple-areolar complex must be established [12].

Special attention must be accorded to patients that previously underwent complementary therapy, such as radio- or chemotherapy and the higher incidence of complications in this particular subgroup must be clearly pointed out before moving onwards $[7,11]$.

Although not uncommon, complications associated with breast reconstructive surgery raised awareness amongst plastic surgeons worldwide, consequently leading to the development of pertinent management options and protocols, with a positive influence on the final outcome for the majority of cases [13].

The purpose of this paper is to describe our personal approach in successfully attempting implant salvage in an irradiated breast reconstruction patient with periprosthetic infection and implant exposure.

\section{Case report}

A 55 year-old, non-smoking, college educated, former breast cancer female patient, presented to our Plastic Surgery Department, in full accordance with the oncological department, requesting a unilateral breast reconstruction procedure. Patient's history revealed a stage IIIB $\left(\mathrm{T}_{4 \mathrm{~b}} \mathrm{~N}_{3 \mathrm{a}} \mathrm{M}_{0}\right)$, ER+/PgR+ (45-50\%/60-65\%), CerbB2+ <10\%, Ki67+30$35 \%$, invasive carcinoma NST affecting the right breast, diagnosed 3 years prior to presentation, for which she underwent a multimodality treatment comprising breast removal surgery and axillary dissection (Madden's modified radical mastectomy), followed by radio-, chemo- and hormonotherapy protocols.

Physical examination upon first admission revealed a normal weight woman (BMI=23.6), with localised adipose tissue deposits, presenting with a type 2 Fitzpatrick skin phototype. No signs of upper limb lymphedema or mobility infringements were noted, neither any scapula alata deformity.
Local inspection showed a flat, pale, 13 $\mathrm{cm}$ oblique scar descending from the anterior axillary fold to the median line, on the anterior part of the right hemi thorax. A slightly visible dog-ear deformity was noted on the scar's medial aspect (Figure 1).

A discrete hyperchromia concerning the scar-facing sides of the mastectomy skin flaps was perceived, along with the slightly elevated railroad tracks signs that followed former suture placement. Local dehydration was unapparent but a faintly decrease in flap trophicity with repercussions upon skin elasticity was pointed out.

Moderate breast hypertrophy associating grade IV ptosis was encountered in the contralateral breast. No palpable axillary or breast masses were observed.

After having walked the patient trough the possibilities of breast reconstruction, in terms of both technical execution and surgical stress, with a good understanding of the potential underlying benefits and risks, the patient opted for a two-stage expander-implant reconstruction.

Therefore, in July 2014 we proceeded with the first stage of the reconstructive procedure. After preoperative markings were performed, under general anaesthesia and intravenous antibiotic prophylaxis, the patient was properly draped and placed in the prone position. Once the mastectomy scar was appropriately excised and sent for histological evaluation, an adequate sub pectoral pocket was carefully dissected in order to accommodate a $350 \mathrm{~cm}^{3}$ Medium Height Style 6200 Mentor Siltex ${ }^{\circledR}$ Breast Expander equipped with an integrated injection port system. Elevating the serratus anterior fascia, which was afterwards attached to the lateral edge of the pectoralis major muscle, covered the infero-external aspect of the expander.

During surgery the trophicity of the skin flaps was thoroughly assessed and deemed suitable for initiating intraoperative expansion of the device (up to $30 \%$ of its rated volume). The resulting surgical defect was managed through a tension free multi-layered direct closure following the careful positioning of suction drains, which were left in place for 7 days, until daily drainage was less than $30 \mathrm{ml}$. No clinical findings indicating flap suffering 
were encountered 2 weeks after the procedure, which lead to the first out of four sessions of postoperative expansion, each separated by a 2-week interval in-between. Final expander volume exceeded its nominal capacity by $10 \%$.
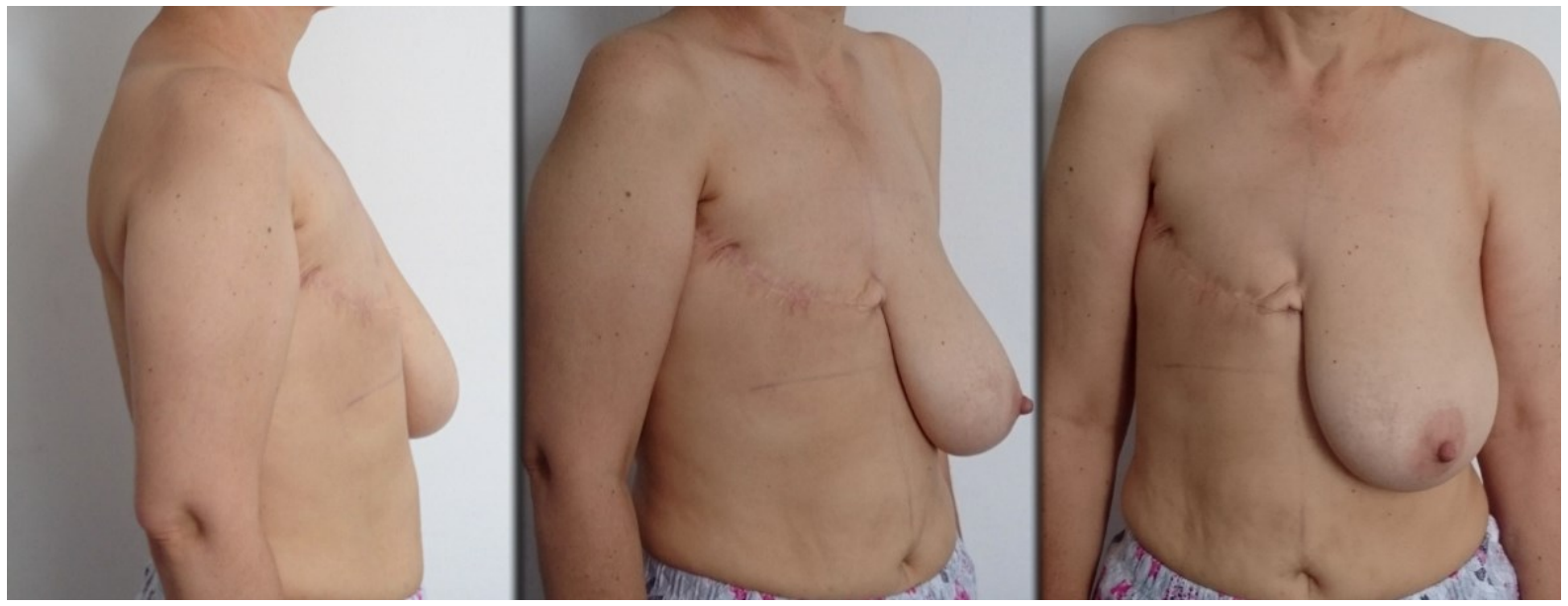

Fig. 1. A flat, pale, $13 \mathrm{~cm}$ oblique scar descending from the anterior axillary fold to the median line is observed on the anterior part of the right hemi thorax. A slightly visible dog-ear deformity is noted on the scar's inner aspect. Discrete hyperchromia concerning the scar-facing sides of the mastectomy skin flaps is perceived, along with the slightly elevated railroad tracks signs that followed former suture placement. Contralateral breast is moderately hypertrophic associating grade IV breast ptosis.

The second stage of the reconstructive procedure was carried out in December 2014, 6 weeks after the last session of postoperative expansion (Figure 2). The breast expander was replaced with a Mentor $345 \mathrm{~cm}^{3}-323$ CPGTM, Cohesive IIITM, Medium Height, Full profile breast implant, following a full circumferential capsulotomy. Intravenous postoperative antibiotic therapy was pursued for 7 days - (Ceftriaxone $1 \mathrm{~g}$ q.d.). Suction drains were kept in place for 6 days, until final output was under $30 \mathrm{ml} /$ day. Postoperative evolution was favourable.

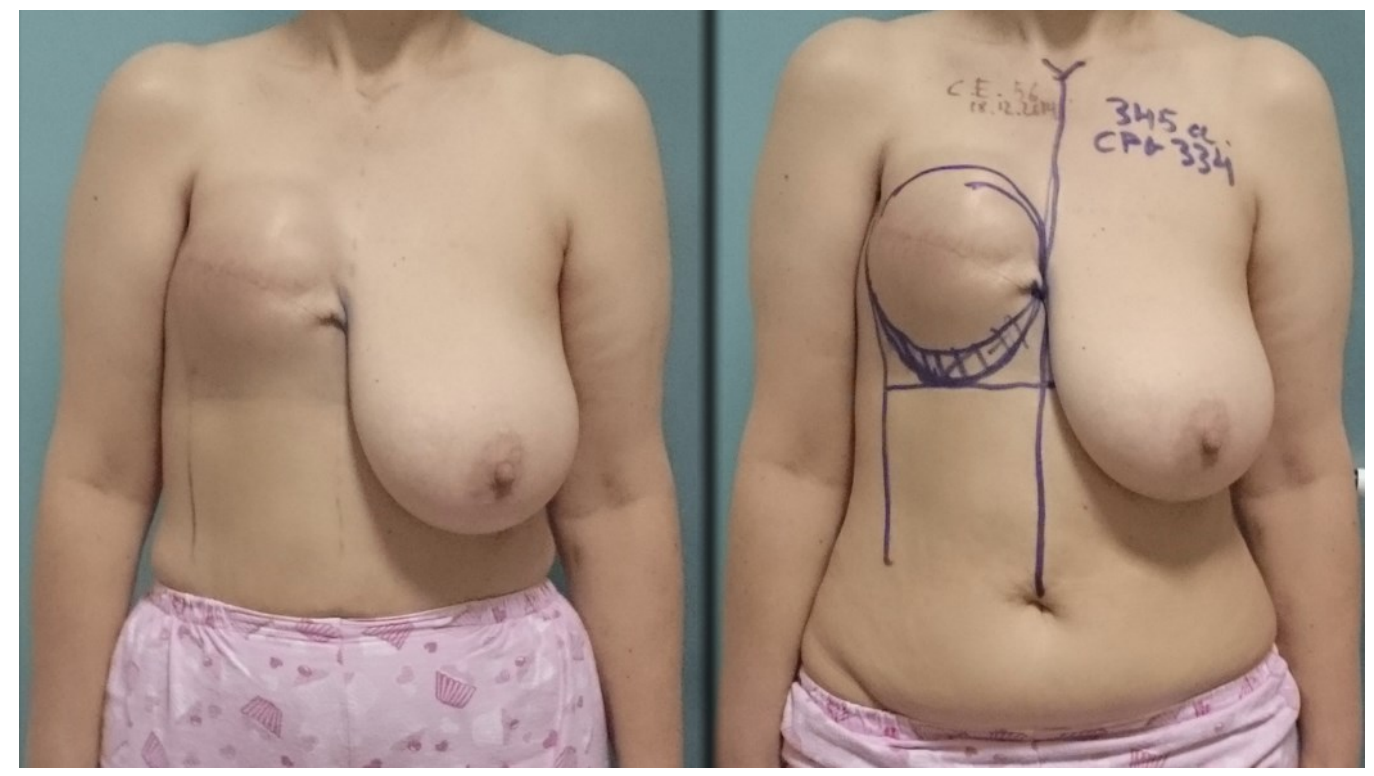

Fig. 2. Preoperative drawings before undergoing permanent breast expander replacement with a Mentor $345 \mathrm{~cm}^{3}$ - 323 CPGTM, Cohesive IIITM, Medium Height, Full profile breast implant. 
After 6 weeks of uneventful evolution, the patient presented to the emergency room with sudden onset cellulitis on the reconstructed breast. Although afebrile, local warmth, mild pain and a minimal periprosthetic seroma were encountered during palpation. Wound dehiscence and minor implant exposure $(0.5$ $\mathrm{cm}^{2}$ ) with subsequent clear, inodorous fluid expression were noted (Figure 3). Blood tests revealed minor leukocytosis 11000 cells $/ \mathrm{mcL}$ with an abnormal elevation in the neutrophil count. Wound cultures were taken and empiric antibiotic therapy was initiated (Ceftriaxone $1 \mathrm{~g}$ q.d.) and later on adapted according to the bacteriological results (Staphylococcus epidermidis. - Ciprofloxacin $1 \mathrm{~g}$ b.i.d. for a 3week period).

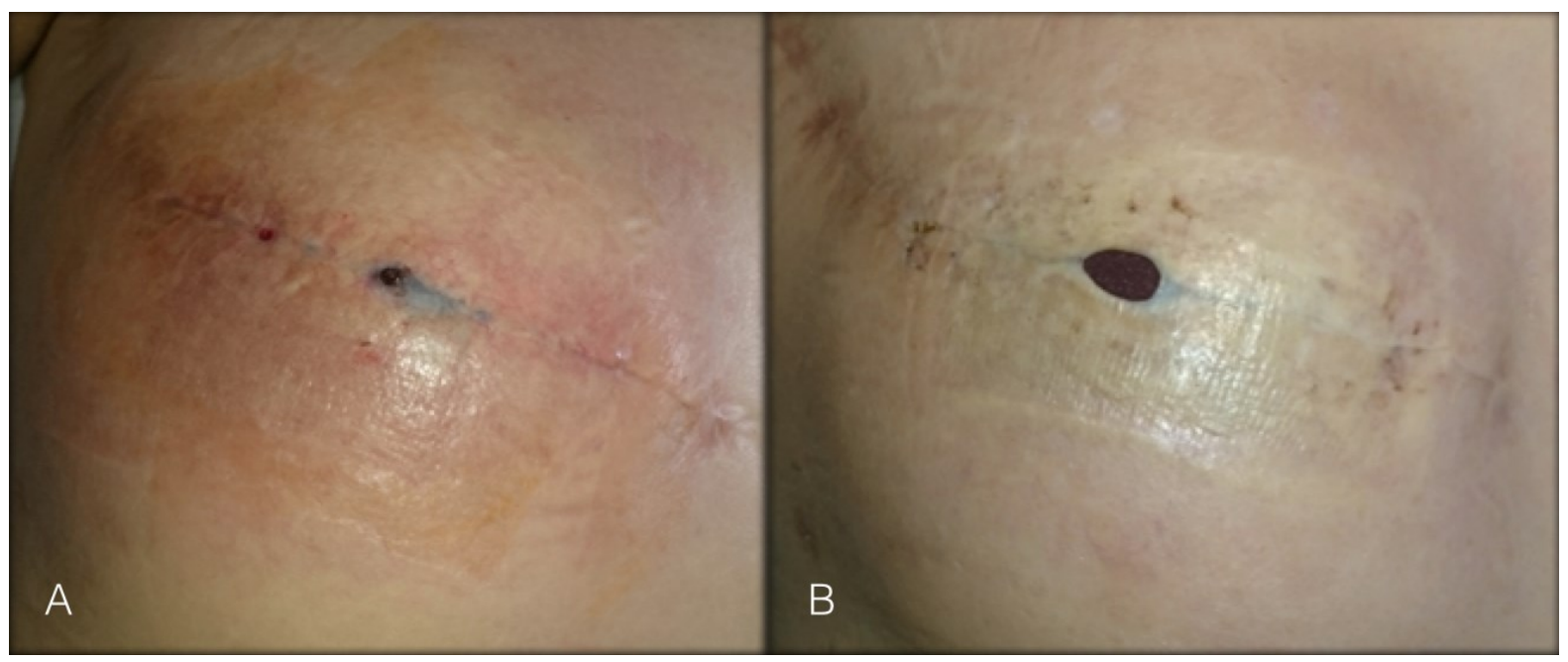

Fig. 3. A. A slightly delineated large area of cellulitis over the reconstructed breast is seen. Wound dehiscence and minor implant exposure $\left(0,5 \mathrm{~cm}^{2}\right)$ are noted. B. Resolution of the inflammatory process occurring after adapted antibiotic therapy. Significant flap thinning and implant exposure are observed.

The satisfactory evolution, both in terms of local conditions and WBC, led to implant replacement, preceded by proper cavity exploration, intra-operative culture takings, copious pulse lavage irrigation (Diluted Povidone-lodine and Ceftriaxone/Gentamicin irrigation solution) and capsulotomy. Postoperative follow-up at 3 weeks exposed no inflammation, local infection or wound dehiscence signs whatsoever, thus allowing for safe suture removal.

Six weeks after, local findings revealed severely thinned mastectomy flaps with the imminent chance of implant exposure. No signs of local or systemic infection were perceived. Blood tests were within normal range and wound cultures were found negative.

Under given circumstances the possibilities of simultaneously achieving implant salvage and providing it with an adequate viable coverage was discussed. Our initial attempt to address this matter consisted in expander pocket enlargement, which was carried out through additional dissection and flap readvancement. Post-operative results after 4 weeks revealed impaired wound healing signs leading to wound dehiscence.

Therefore we proceeded with the elevation of a $6 \times 12 \mathrm{~cm}$ skin isle latissimus dorsi myocutaneous flap designed to attain the upcoming resulting defect (Figure 4).

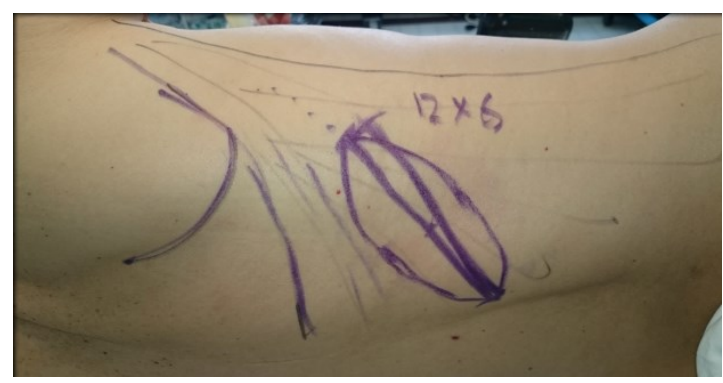

Fig. 4. Preoperative drawings designed for a $6 \times 12$ $\mathrm{cm}$ skin isle latissimus dorsi myocutaneous flap harvest. 
During dissection, the thorocodorsal pedicle was found to have been severed thus rendered futile for flap nourishment. Although the classical vascular pattern of the flap was altered, intra-operative evaluation of the latissimus dorsi muscle displayed a substantial anastomotic vascular loop with the vessels providing serratus anterior muscle, which allowed us to resume the procedure.

Significant flap venous congestion, comprising the medial half of its skin isle, occurred on postoperative day 3 and gradually led to substantial flap necrosis, which was conservatively treated for a month's time (Figure 5). No signs of infection were observed.

Intra-operative debridement of all compromised tissue was subsequently carried out resulting in a nearly $100 \mathrm{~cm}^{2}$ defect, which was addressed by means of a fasciocutaneous loco-regional perforator flap, based on the first encountered superior epigastric artery perforator (Figure 6), preoperatively identified through infrared thermal imaging (Figure 7).

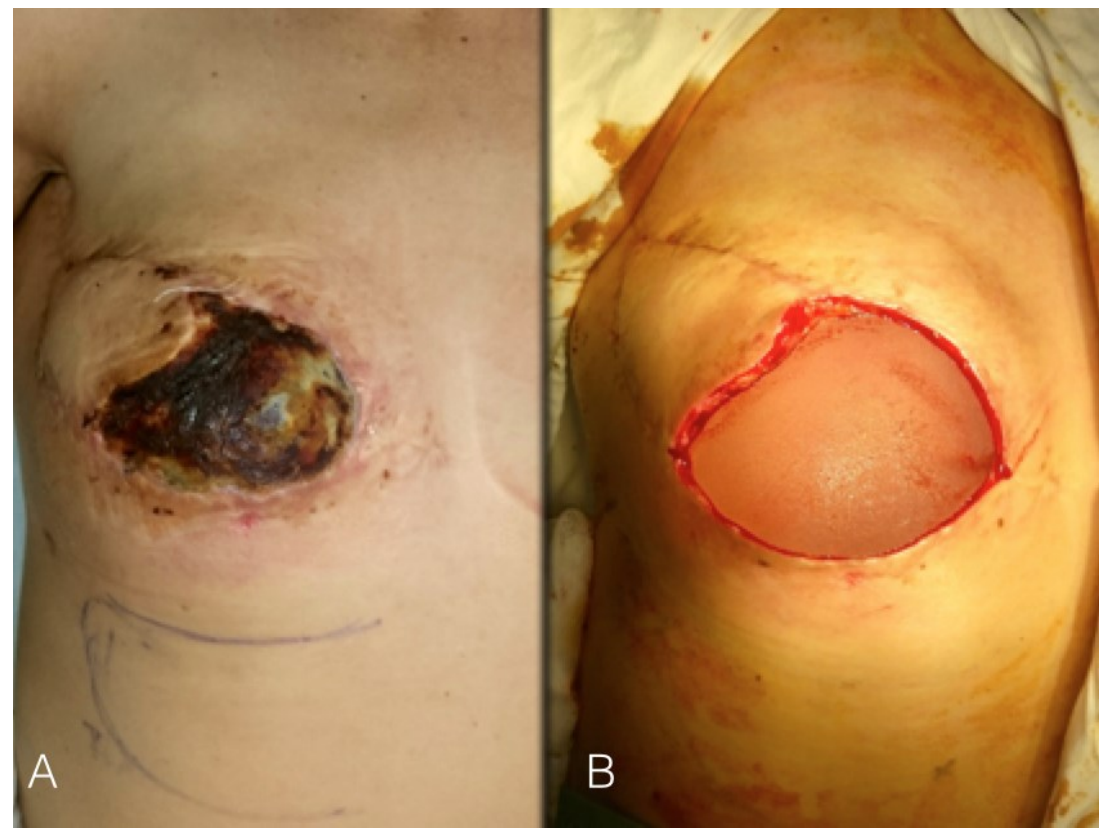

Fig. 5 A. Significant flap necrosis comprising the medial half of its skin isle. No signs of infection are identified.B. Intra-operative debridement of all compromised tissue leading to a nearly $100 \mathrm{~cm}^{2}$ defect

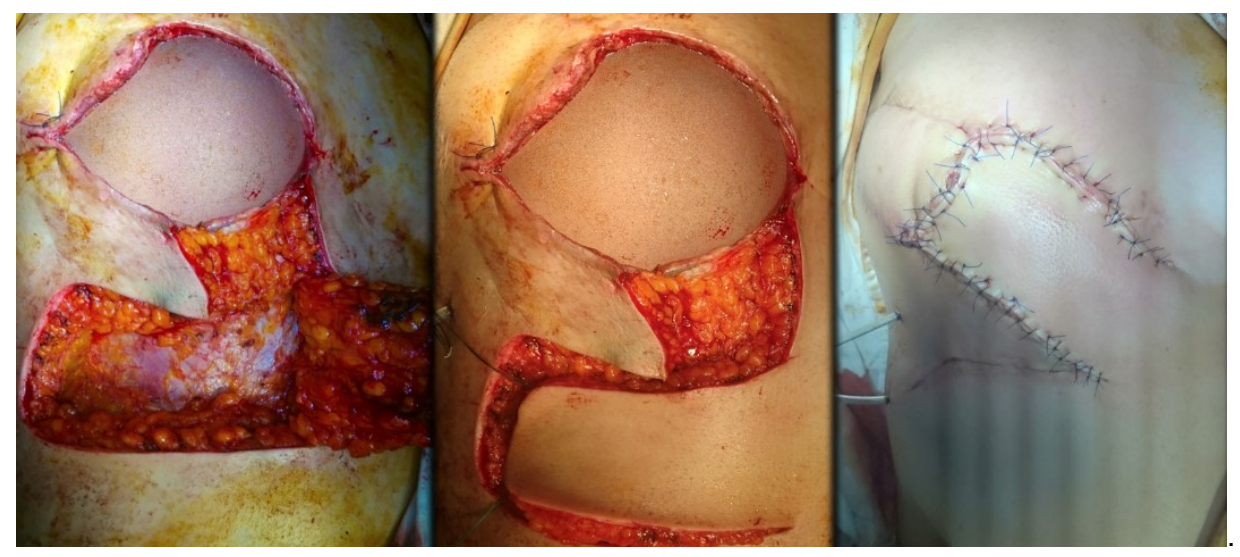

Fig. 6. A Fasciocutaneous loco-regional perforator flap design, based on the first encountered superior epigastric artery perforator identified pre-operatively through infrared thermal imaging. 


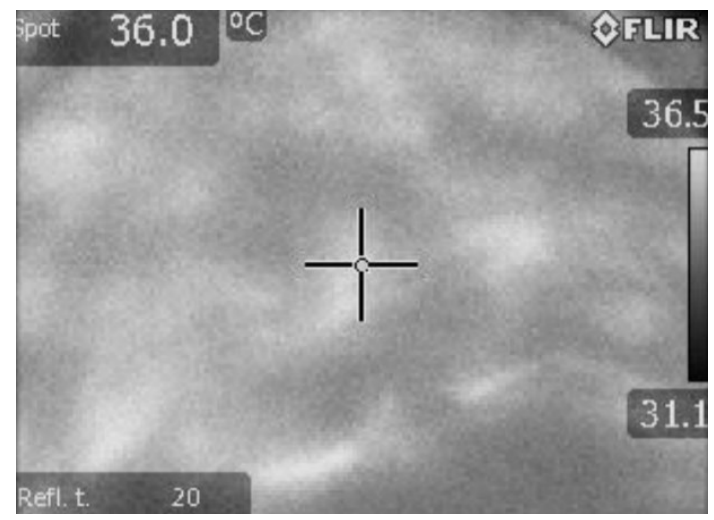

Fig. 7. Infrared thermal image depicting a strong hot spot (cross) indicating a reliable vascular source emerging from the superior epigastric artery upon which flap elevation is deemed possible.

The device was removed and properly placed into a diluted betadine solution whilst the resultant cavity was thoroughly irrigated. Next, the implant was repositioned into the submuscular pocket and adequate coverage was achieved through flap translation. Postoperative evolution was uneventful with stable results encountered at 3 months followup. The reconstructive procedure will be resumed 6 months after surgery when nippleareolar complex reconstruction and contralateral symmetrisation mammaplasty are envisaged (Figure 8).

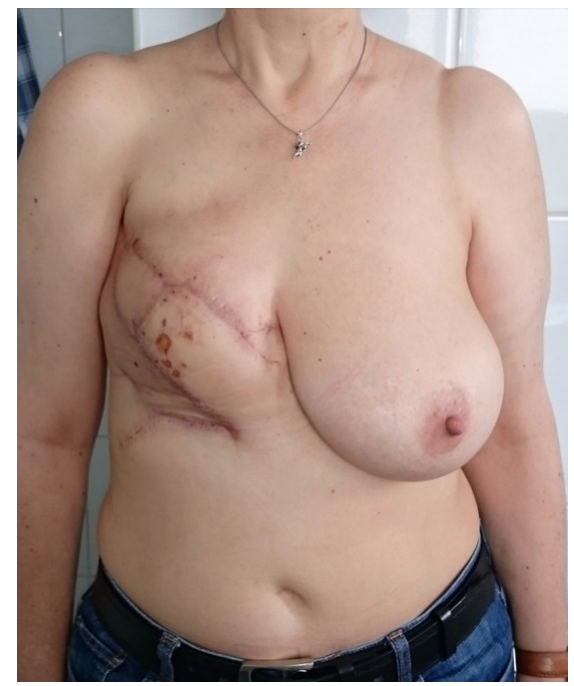

Fig.8. Postoperative result prior to contralateral symmetrisation mammaplasty and nipple-areolar complex reconstruction. Minor blisters caused by adhesive skin closures are noted on the flap's cranial aspect.

\section{Discussion}

Currently acknowledged as a "coup de grâce" regarding breast cancer management, breast reconstructive procedures have managed to impress both through their remarkable results but also through the vast display of procedural variants and through their degree of technical complexity [14, 15].

Recent advancements in the alloplastic material industry [16] altogether with significant improvements achieved in the microsurgical field allow for a great deal of flexibility when it comes to choosing how to proceed on the reconstructive path [17].

Present-day breast reconstruction guidelines advocate patient selection as a key factor in attaining the best possible outcomes and overall success [7, 11]. Patient-related features (clinical aspects, history of complementary therapy and level of expectations) as well the surgeon's experience and capability to execute the envisaged reconstructive procedures stress out the important degree of practical difficulty associated with this type of surgery $[9,11]$.

Therefore, upon initial evaluation, our patient was deemed suitable for an autologous breast reconstruction procedure. Previous history of chest wall radiotherapy (44 Gy delivered in 22 fractions throughout a 5-week period), although well tolerated, without any alarming modifications concerning flap trophicity or skin structure combined with the patient's clinical characteristics (increased contralateral breast volume, localised abdominal fat deposits, lack of any abdominal scarring, non-smoking status and general good health) led to our understandable proposal for an abdomen-based breast reconstruction.

Despite having a more appropriate profile for this type of surgery [7, 11, 18], patient's preferences towards a rapid recovery, no additional scarring and less complicated intervention made us consider a prostheticbased reconstructive procedure.

Our previous experience in this particular matter, in terms of complications and results, tended to coincide with that of several major breast reconstruction centers worldwide $[7,13$, 19-21]. 
Prince et al, in a retrospective study conducted on 43 patients who underwent attempted prosthesis salvage, encountered $76.7 \%$ success rate. Among their patients, previous radiotherapy was not identified as a statistically significant factor associated with poorer salvage rate [22]. This particular finding is supported by the similar data provided by Yii and Khoo [23], by Spear and Seruya [24] and by the Michigan breast reconstruction outcome study [25]. On the other hand certain pathogens such as $S$. epidermidis [22] or $S$. aureus, Gram-negative rods and Candida parapsilosis [24] were associated with salvage failure in a statistically significant manner, which led these authors to consider them as relative contraindications to implant salvage.

McCarthy et al, in a retrospective comparative study conducted on 1170 expander/implant patients, found that implant loss was statistically correlated with a BMI > 30 and with an active smoking status. An overall complication rate of $15 \%$ was encounter in this study, with $2.5 \%$ of the patients having the implant/expander removed [26].

Hirsch et al, in their retrospective review of 876 patients who underwent expander/implant breast reconstruction, found that during the expander stage a BMI over 30 was statistically correlated with the development of complications leading to explantation or conversion to flap reconstruction whilst during the implant stage, a history of previous radiotherapy was proven relevant to developing complications. Age $>50$, smoking within the past month and a history of premastectomy or postmastectomy irradiation were found to be the only risk factors that had a statistically significant effect on the development of complications during both stages [27].

Reish et al, in a retrospective review, conducted on 94 prosthetic-based breast reconstruction patients admitted for possible or definitive infection, found a total salvage rate with antibiotics and/or immediate operative exchange of the implant in $37.3 \%$ of the cases. Implant salvage failure in the rest $62.7 \%$ of the patients was correlated with a higher average WBC count upon admission and with a more likely methicillin resistant Staphylococcus aureus infection [28].

The modern low dosage breast radiation therapy ensued by a substantial 25-month procedural-free interval, hence favoring local tissular regeneration [28, 29], altogether with the unapparent radiation-induced skin damage signs over the mastectomy flaps, made our patient also eligible for a two stage alloplastic procedure.

Although it has been postulated that fractionate radiotherapy also deals damage to the adjoining healthy tissue, it does so in a sublethal fashion, thus allowing for optimal cellular restoration [29, 30]. However, current understanding with regard to molecular interactions and signaling pathways is yet to be fully understood, rendering it difficult to explain postoperative complications such as impaired wound healing $[21,29]$.

When facing clinical periprosthetic infection signs and/or exposure, a priori implant salvage should be attempted [22]. Modern practice recurs to device explantation as a last resort, namely when dealing with rebellious infections or when patients decide to abort the reconstructive procedure [31, 32].

Unanimously-approved specific protocols relative to these complications are not current available, yet several valid management options revolving around systemic antibiotic therapy, conservative treatment, direct wound closure, device replacement with direct closure or device replacement with adequate muscle coverage provide a comprehensive array of salvage procedures [22, 32, 33].

Achieving implant coverage by means of a pedicled latissimus dorsi myocutaneous flap has proven extremely reliable for both breast reconstruction and implant salvage procedures [19].

Pivoting on its dominant pedicle, this type $\checkmark$ muscle flap [34] significantly improves the tissue quality overlying the prosthesis, specifically on its lower pole, with a positive impact on the final aesthetic result and on implant exposure recurrence $[19,35]$.

Although deprived of its thoracodorsal pedicle, our flap was considered viable owing not only to the impressive size of the aforementioned vascular anastomotic loop but 
also to the pulsatile bleeding pattern witnessed after detaching its distal fibres.

We therefore hold the insufficient venous drainage, which, prior to disinsertion was mainly assured by the posterior intercostal veins, responsible for flap congestion and its subsequent failure in the distal half.

When aiming to accomplish defect coverage by employing local pedicled flaps, proper preoperative evaluation of the underlying vascular pattern is mandatory [36, 37, 38].

In our experience, thermal imaging assessment has been very effective in terms of perforator interception, hence simplifying the decision making process and easing dissection.

Use of the angiosome concept in flap tailoring [39], offers the best possible postoperative results by providing the maximum amount of tissue to be harvested in a relatively safe fashion.

\section{Conclusions.}

Presently integrated in breast cancer management protocols, breast reconstruction procedures represent a step forward in restoring a woman's self-perception as a whole, having great impact on social functioning and on the quality of life.

Although patient selection plays a crucial role in attaining the best possible reconstructive results, the decision on how to proceed on the reconstructive path relies on doctor-patient mutual agreement, once proper

\section{References}

1. Jecan CR, Hernic AD, Filip IC, Raducu L. Clinical data related to breast reconstruction: looking back on the 21th century and forward to the next steps. JMMS 2015; II(1):34e-42e.

2. Trabulsy PP, Anthony JP, Mathes SJ. Changing trends in postmastectomy breast reconstruction: a 13-year experience. Plast Reconstr Surg 1994; 93(7):1418-27.

3. Zhong $\mathrm{T}$, McCarthy CM, Price AN, Pusic AL. Evidence-based medicine: breast reconstruction. Plast Reconstr Surg 2013; 132(6):1658-69. subject-related information is provided to the latter.

Even if radiation-induced skin damage may be clinically unapparent, special caution must be exercised when solely prostheticbased reconstructive procedures are to be engaged, rendering autologous procedures or combined approaches more suitable in these cases.

When it comes to preoperative perforator assessment and flap planning, thermal imaging has proven to be a valuable tool, significantly increasing both flap's bulk and also its chances of survival.

Implant salvage is not only possible but also the correct attitude to be adopted when dealing with clinical signs of periprosthetic infection or when device exposure is imminent.

In addition to all the foregoing, patient guidance and support should be provided systematically during the entire extent of the reconstructive process, providing adequate information and allowing these women to surpass their anxieties and doubts regarding the ultimate result. axillary lymph nodes.

\section{Consent}

Written informed consent was obtained from the patient for publication of this case report and accompanying images.

\section{Conflict of interest}

The author(s) declare that they have no competing interests.
4. Serletti JM, Fosnot J, Nelson JA, Disa JJ, Bucky LP. Breast reconstruction after breast cancer. Plast Reconstr Surg 2011; 127(6):124e-35e.

5. Kronowitz ST. State of the art and science in breast reconstruction. Plast Reconstr Surg 2015; 135(4):755e-71e.

6. Harmer V. Breast reconstruction: one size does not fit all. Br J Hosp Med 2015; 76(4):209-10.

7. Disa JJ, McCarthy CM, Mehrara BJ, Pusic AL, $\mathrm{Hu}$ QY, Cordeiro PG. Postmastectomy reconstruction: an approach to patient 
selection. Plast Reconstr Surg 2009; 124(1):4352.

8. Spear SL, Spittler CJ. Breast Reconstruction with implants and expanders. Plast Reconstr Surg 2001; 107(1):177-87.

9. Sigurdson L, Lalonde DH. Breast Reconstruction Plast Reconstr Surg 2008; 121(1 Suppl):1-12.

10. Heller L, Miller MJ. Patient education and decision making in breast reconstruction. Semin Plast Surg 2004; 18(2): 139-147.

11. Nahabedian MY. Breast reconstruction: a review and rationale for patient selection. Plast Reconstr Surg 2009; 124(1):55-62.

12. Revol M, Binder J-P, Danino A, May $P$, Servant J-M. Manuel de chirurgie plastique, reconstructice et esthétique $2^{\text {éme }}$ edition. Montpellier: Sauramps Medical, Septembre 2012.

13. Slezak S. An evidence-based approach to breast reconstruction. Plast Reconstr Surg 2010; 126(6):2177-83.

14. Morrow M. Trends in the surgical treatment of breast cancer. Breast $J$ 2010; 16 (Suppl 1):S17-9.

15. Beier JP, Horch RE, Bach AD. Breast reconstruction after breast- cancer surgery. $N$ Engl J Med 2009; 360(4):418-9.

16. Jecan $C R$, Filip $\mathrm{Cl}$, Hernic $A D$, Raducu L. Advanced polymeric biomaterials - an integrated solution for plastic, reconstructive and aesthetic surgery. Key Engineering Materials 2015; 638 (2015):197-204.

17. Yu SC, Kleiber GM, Song DH. An algorithmic approach to total breast reconstruction with tissue transfer. Plast Surg 2013; 40(3):173-180.

18. Platt J, Baxter $N$, Zhong $T$. Breast reconstruction after mastectomy for breast cancer. CMAJ 2011; 183(18): 2109-2116.

19. Spear SL, Boehlmer JH, Taylor NS, Prada C. The role of the latissius dorsi flap in reconstruction of the irradiated breast. Plast Reconstr Surg 2007; 119(1): 1-9.

20. Momoh AO, Hons RA, Kelley BP, Aliu O, Kidwell KM Kozlow JH. A systematic review of complications of implant-based breast reconstruction with prereconstruction and postreconstruction radiotherapy. Ann Surg Oncol 2014; 21:118-124.

21. Kearney AM, Brown MS, Soltanian HT. Timing of radiation and outcomes in implant-based breast reconstruction. J Plast Reconstr Aesthet Surg 2015; 68(12):1719-26.

22. Prince MD, Suber JS, Aya-ay ML, et al. Prosthesis salvage in breast reconstruction patients with periprosthetic infection and exposure. Plast Reconstr Surg 2012; 129(1):42-8129.
23. Yii NW, Khoo CT. Salvage of infected expander prostheses in breast reconstruction. Plast Reconstr Surg 2003; 111:1087- 1092.

24. Spear SL, Seruya M. Management of the infected or exposed breast prosthesis: A single surgeon's 15-year experience with 69 patients. Plast Reconstr Surg 2010; 125:10741084.

25. Alderman AK, Wilkins EG, Kim HM, Lowery JC. Complications in postmastectomy breast reconstruction: Two-year results of the Michigan Breast Reconstruction Outcome Study. Plast Reconstr Surg 2002; 109:22652274.

26. McCarthy C, Mehrara B, Riedel E, et al. Predicting complications following expander/implant breast reconstruction: An outcomes analysis based on preoperative clinical risk. Plast Reconstr Surg 2008; 121:1886-1892.

27. Hirsch EM, Seth AK, Kim JY, et al. Analysis of risk factors for complications in expander/implant breast reconstruction by stage of reconstruction. Plast Reconstr Surg 2014; 134(5):692e-699e.

28. Reish RG, Damjanovic B, Austen WGJ et al. Infection following Implant-based reconstruction in 1952 consecutive breast reconstructions: salvage rates and predictors of succes. Plast Reconstr Surg 2013; 131(6):1223-30.

29. Hubenak JR, Zhang Q, Branch CD, Kronowitz SJ. Mechanisms of injury to normal tissue after radiotherapy: a review. Plast Reconstr Surg 2014; 133(1):49e-56e.

30. Peled AW, Foster RD, Esserman LJ, Park CC, Hwang ES, Fowble B. Increasing the time to expander-implant exchange after postmastectomy radiation therapy reduces expander-implant failure. Plast Reconstr Surg 2012; 130(3):503-9.

31. Rabey NG, Malata CM. Succesful salvage of exposed breast implants in previously irradiated patients using local fasciocutaneous flaps. Eur J Plast Surg 2013; 36:195-198.

32. Spear SL, Howard MA, Boehmler JH, Ducic I, Low M, Abbruzzesse MR. The infected or exposed breast implant: management ant treatment strategies. Plast Reconstr Surg 2004; 113(6):1634-44.

33. Wilkinson TS, Swartz BE, Toranto IR. Resolution of late-developing periprosthetic breast infections without prosthesis removal. Aesth Plast Surg 1985; 9:79-85.

34. Mathes SJ, Nahai F. Classification of the vascular anatomy of muscles: experimental and clinical correlation. Plast Reconstr Surg 
1981; 67(2):177-87.

35. De Mey A, Lejour M, Declety A, Meythiaz AM. Late results and current indications of latissimus dorsi breast reconstructions; Brit $J$ Plast Surg 1991, 44(1):1-4.

36. Sheena $Y$, Jennison $T$, Hardwicke JT, Titley OG. Detection of perforators using thermal imaging. Plast Reconstr Surg 2013; 132(6):1603-10.

37. Bagavathiappan S, Saravanan T, Phillip J, Jayakumar T, Raj B, Karunanithi R. Infrared thermal imaging for detection of peripheral vascular disorders. J Med Phys 2009; 34(1):
43-47.

38. Mercer JB, Weerd L, Miland ÅO, Weum S. Pre, intra-, and postoperative use of dynamic infrared thermography (DIRT) provides valuable information on skin perfusion in perforator flaps used in reconstructive surgery. FLIR Technical Series, Application note for Research \& Science, 2011 FLIR Systems, Inc. [http://www.flir.jp/WorkArea/DownloadAsset.as px?id=50050. available at 12/27/2015]

39. Taylor GI, Palmer JH. Angiosome theory. JPRAS 1992; 45(4):327-328. 